\title{
Prevalence of obesity and factors associated with it in a worksite setting in Malaysia.
}

\begin{abstract}
Socio-economic status, lifestyle behaviors, and psychosocial factors have been implicated in the development of overweight and obesity. This study aims to observe the prevalence of overweight and obesity in an academic worksite and to examine the possible association between variables such as socio-economic characteristics, work factors, psychosocial factors, and weight control behaviors and obesity. In this study, the target population were full-time academic and non-academic staff. Body mass index (BMI) and waist circumference (WC) were computed to determine obesity. A pretested self-administered questionnaire was used to obtain information on socio-demographic factors, work related factors, psychosocial factors, and weight control behaviors. Data were obtained on 367 adults of whom 39.2\% were males and $60.8 \%$ females. Overweight was seen in $31.9 \%$ of males and $26.5 \%$ of females while $16.1 \%$ of them were obese, irrespective of gender. Central obesity was noted in about $37 \%$ of males and $39 \%$ of females. The results showed that socio-demographic factors (age, gender, and education) and psychosocial factors (perceived health status, body weight perception, and weight-control goals) were significantly associated with BMI. Working hours were also significantly associated with BMI. However, weight control practices (diet-control practices and physical activity practices) were not significantly associated with BMI. In conclusion, this study found a higher prevalence of overweight and obesity among employees of a selected public university in comparison to the general population. Socio-demographic, psychosocial factors, and working hours were found to contribute to obesity in this sample of adults.
\end{abstract}

Keyword: Behavioral factors; Obesity; Psychosocial factors; Work factors; Worksite setting. 